\title{
Cognitive development in children with 22q11.2 deletion syndrome
}

Sasja N. Duijff, Petra W. J. Klaassen, Henriette F. N. Swanenburg de Veye, Frits A. Beemer, Gerben Sinnema and Jacob A. S. Vorstman

\section{Background}

People with 22q11.2 deletion syndrome (velo-cardio-facial syndrome) have a 30 -fold risk of developing schizophrenia. In the general population the schizophrenia phenotype includes a cognitive deficit and a decline in academic performance preceding the first episode of psychosis in a subgroup of patients. Findings of cross-sectional studies suggest that cognitive abilities may decline over time in some children with 22q11.2 deletion syndrome. If confirmed longitudinally, this could indicate that one or more genes within $22 q 11.2$ are involved in cognitive decline.

\section{Aims}

To assess longitudinally the change in IQ scores in children with 22q11.2 deletion syndrome.

\section{Method}

Sixty-nine children with the syndrome were cognitively assessed two or three times at set ages 5.5 years, 7.5 years and 9.5 years.

\section{Results}

A mean significant decline of 9.7 Full Scale IQ points was found between ages 5.5 years and 9.5 years. In addition to the overall relative decline that occurred when results were scored according to age-specific IQ norms, in 10 out of a group of 29 children an absolute decrease in cognitive raw scores was found between ages 7.5 years and 9.5 years. The decline was not associated with a change in behavioural measures.

\section{Conclusions}

The finding of cognitive decline can be only partly explained as the result of 'growing into deficit'; about a third of 29 children showed an absolute loss of cognitive faculties. The results underline the importance of early psychiatric screening in this population and indicate that further study of the genes at the 22q11.2 locus may be relevant to understanding the genetic basis of early cognitive deterioration

\section{Declaration of interest}

None.
Children with $22 \mathrm{q} 11.2$ deletion syndrome are reported to have learning difficulties and are at a greater risk of psychiatric disorders including autism-spectrum disorders and attentiondeficit hyperactivity disorder. During adolescence and early adulthood, up to $30 \%$ of people with the syndrome develop schizophrenia, thus suggesting that the 22q11.2 deletion can be considered the highest known genetic risk factor for schizophrenia other than having a monozygotic twin sibling with the illness. ${ }^{1-5}$ Several cross-sectional studies have found a negative correlation between age and IQ scores in 22q11.2 deletion syndrome, ${ }^{6-10}$ suggesting that at least some of these individuals show a gradual decline in cognitive development as they grow into adulthood. Results of two longitudinal studies, respectively in adolescents and in patients with 22q11.2 deletion syndrome in late childhood/ adolescence, are consistent with this suggestion. ${ }^{11,12}$ There may be several explanations for these observations. The normative scale might have become more stringent with the introduction of a revised version of the scale to compensate for the 'Flynn effect', an upward drift of approximately 3 points per decade in normative performance on IQ tests. ${ }^{13}$ Alternatively, adolescents with weaknesses in the area of abstract reasoning might 'grow into deficit', meaning that a decrease in IQ scores with age is not indicative of an absolute decline in cognition, but rather parallels an inability to adapt to the gradual increase in the level of cognitive requirements with age. Although these factors may play a part in the presumed decline in cognitive abilities in young people with the 22q11.2 deletion, the high prevalence of schizophrenia in this syndrome strongly suggests that there might be an alternative explanation. Research into cognitive development in schizophrenia in the general population indicates that cognitive decline occurs in late adolescence, ${ }^{14}$ but some studies also suggest that academic or cognitive deficits can be found as early as in the first grade of school. ${ }^{15-18}$ These findings are in keeping with the neurodevelopmental model of schizophrenia. Against this background, a cognitive decline in people with 22q11.2 deletion syndrome - in this study defined as a decrease in IQ scores shown by repeated measurements may be viewed as the first manifestation of schizophrenia. Indeed, in a cross-sectional study of 43 children with $22 \mathrm{q} 11.2$ deletion syndrome, Debbané et al reported that children with psychotic symptoms had significantly lower verbal IQ (VIQ) scores than those without such symptoms. ${ }^{19}$ Gothelf et al reported that a decline in VIQ in adolescence may be associated with an increased risk of psychotic symptoms during follow-up. ${ }^{11,20}$ In an attempt to replicate these findings and predict psychotic symptoms in 22q11.2 deletion syndrome, Antshel et al reported a positive association between deterioration of several cognitive functions, in particular executive and verbal abilities, and the development of prodromal symptoms in late childhood/adolescence. ${ }^{12}$ In the largest cross-sectional study to date $(n=172)$, a negative correlation between age and Full Scale IQ (FSIQ) was reported, with a stronger negative correlation between VIQ subscales and age compared with performance IQ (PIQ). ${ }^{8}$ The authors noted that the cross-sectional design of their study was a limitation and suggested that in future longitudinal studies 'the use of raw scores, in addition to age-normed scores, should be used to help assess cognitive change over time. ${ }^{8}$

Given the increased risk of schizophrenia in people with 22q11.2 deletion syndrome and the observed premorbid deficit in cognitive abilities associated with schizophrenia in the general 
population, the study of cognitive development in young children with the 22q11.2 deletion is highly relevant. If, as research suggests, cognitive abilities decline with age in these children, this implies that one or several genes within the 22q11.2 region are causally related to cognitive deterioration during childhood. Second, this study may clarify at what age this cognitive decline can be observed, and - perhaps more importantly - whether all children display this decline or alternatively only a subgroup. The latter question is particularly relevant and could be a focus of subsequent studies, as only a subgroup of children with the deletion syndrome go on to develop schizophrenia later in adolescence or adulthood. The objective of this prospective longitudinal study was to assess changes in IQ levels in children between the ages of 5.5 years and 9.5 years. To investigate whether any observed decrease in IQ score was due to insufficient cognitive development leading to an increasing discrepancy with agerequired norms (growing into deficit), or rather a result of an absolute decline in cognitive capabilities, both subtest scaled scores (i.e. age-specific normative scores) and subtest raw scores (absolute test scores, prior to age-normative adjustment) were analysed. It is important to note that although we included global measures of behaviour, the study was not designed to assess neuropsychiatric symptoms, in particular the onset of psychotic symptoms.

\section{Method}

The study was part of a nationwide prospective longitudinal psychological study of IQ levels and behaviour in children with 22 q11.2 deletion syndrome. In this ongoing study, enlistment is allowed at any age between 1 year and 15 years. Participants were recruited through referrals from genetic counsellors, cleft palate clinics and paediatric cardiologists from hospitals throughout The Netherlands or through postings on the website of the Dutch parent support group VCFS/22q11 (www.vcfs.nl). The inclusion criterion was the presence of a 22q11.2 deletion confirmed by fluorescence in situ hybridisation (FISH) analysis or multiplex ligation-dependent probe amplification. ${ }^{21}$ The assessment protocol was approved by the Dutch Medical-Ethical Review Commission. Written informed consent was obtained from all parents or guardians.

A total of 77 children (27 boys and 50 girls) were originally included. Of this group, 8 children ( 1 boy, 7 girls) were estimated to have intellectual disabilities below the range of the DSM-IV category 'moderate mental retardation.22 Data pertaining to the latter children were excluded from all statistical analyses because the longitudinal development of IQ levels as assessed by the test protocol could not be monitored (see the online supplement for additional information on this group). Of the remaining group of 69 children ( 26 boys, 43 girls), 41 were assessed twice, and 28 were assessed three times at the ages of 5.5 years, 7.5 years and 9.5 years exactly (Table 1 ). Drop-out rates at various measurement points are also described in Table 1.

\section{Education}

Parents were asked to report their highest level of education attained and were categorised according to the International Standard Classification of Education designed by the United Nations Educational, Scientific and Cultural Organization (UNESCO). ${ }^{23}$ In the general Dutch population, $27 \%$ of men and $24 \%$ of women have completed higher (tertiary) education. ${ }^{24}$ When controlling for the effects of FSIQ with level of parental education, it was found that within the group, $31-34 \%$ of fathers and $31-40 \%$ of mothers had completed tertiary education.

\section{Assessment}

At 5.5 years of age the Dutch version of the Wechsler Preschool and Primary Scale of Intelligence - Revised (WPPSI-R) was used. ${ }^{25}$ However, 15 (of 53) children could not be assessed with this test owing to speech problems; in these cases the revised Snijders-Oomen Non-verbal test for ages 2.5-7 years (SON-R 2.5-7) was used. ${ }^{26}$ Correlations between FSIQ scores derived using the SON-R 2.5-7 and WPPSI-R are high $(r=0.75) .{ }^{26}$

At 7.5 years and 9.5 years of age the children's FSIQ was assessed using the Dutch versions of the Wechsler Intelligence Scale for Children - Revised (WISC-RN) and/or the Wechsler Intelligence Scale for Children Third Edition (WISC-III-NL). ${ }^{27,28}$ The Wechsler tests consist of two scales, one assessing VIQ and the other PIQ. The test thus yields three scores (FSIQ, VIQ and PIQ), each with a mean of 100 and a standard deviation of 15 . The study started before the WISC-III-NL was published in The Netherlands; therefore early participants $(n=15)$ were assessed with the WISC-RN at 7.5 years and with the WISC-III-NL at 9.5 years. All other participants $(n=29)$ were assessed with the WISC-III-NL at both ages. Research on the correlation between WISC-RN and WISC-III-NL suggests that the two tests yield similar results for FSIQ $(r=0.88) .{ }^{28}$ Since changes in cognitive test performance can be associated with changes in behaviour or influenced by the use of psychotropic medication, parents were asked to fill in the Child Behavior Checklist for Ages 6-18 (CBCL 6-18) while the child was being assessed. ${ }^{29}$ Also, use of psychotropic medication was recorded at both assessment points.

\section{Statistical analysis}

In total, 69 participants were successfully assessed two or three times. Descriptive analyses were used. To take missing values into account, the research design chosen was a multilevel analysis by

Assessments
5.5 and 7.5 years
7.5 and 9.5 years
5.5 and 9.5 years
$5.5,7.5$ and 9.5 years
Total per age group
a. No assessments: 2 children declined further participation, 1 moved abroad, 17 not yet aged 9.5 years.
b. No assessments: 15 children not participating in study at this age, 1 with somatic health problems at this age.
c. No assessments: 2 children with somatic health problems, 3 not able to be tested with standard test protocol.


use of mixed models. Mixed models are used for the analysis of data measured over time to study population-level change and individual differences in change characteristics and allow for missing data. The sensitivity of parameter estimates to missing data assumptions can be studied, for example, by fitting multiple models that make different assumptions about the missing data process.

Potentially confounding variables (gender and test used) were controlled for. First, multilevel analyses of the development of FSIQ, VIQ and PIQ scores between 5.5 years and 9.5 years were performed $(n=69)$. Second, given that only the WISC-RN and the WISC-III-NL allow for analysis of the progression of the subtest scores (the WPSSI-R results in different subtest scores), measurement at 5.5 years was discarded and a paired samples $t$-test was used to analyse the subtest scaled scores at 7.5 years and 9.5 years for children assessed with either the WISC-RN or WISC-III-NL $(n=44)$. Third, to differentiate between growing into deficit and the possibility of an absolute decline in cognitive abilities, the results of a subgroup of children who were tested with the WISC-III-NL at both ages 7.5 years and 9.5 years $(n=29)$ were examined using the raw subtest scores. An independent samples $t$-test was used. Finally, a number of statistical tests were compared to exclude the possibility of relevant test-related confounding effects on the results. To compare the results of the various tests used at the different ages (SON-R 2.5-7 and WPPSI-R at 5.5 years; WISC-RN and WISC-III-NL at 7.5 years), the possibility of testrelated effects on the changes in IQ scores over time was examined. This was especially important because the primary interest of this study is the longitudinal development of cognition. To this end, IQ changes were compared between children tested with the WISC-III-NL at both ages $(n=29)$ and those tested with the WISC-RN at 7.5 years and WISC-III-NL at 9.5 years $(n=15)$, using an independent samples $t$-test. Significance level was set at 5\%. All statistical calculations were carried out using SPSS version 15.0 for Windows.

\section{Results}

\section{Cognitive development 5.5-9.5 years}

Table 2 presents the cross-sectional data and gender-specific development in IQ levels for the sample $(n=69)$. Gender differences in favour of girls were found for FSIQ scores at 5.5 years and 7.5 years of age, for VIQ scores at 5.5 years and for PIQ scores at 7.5 years. Multilevel analyses of the cognitive tests indicated that FSIQ scores declined significantly $(P<0.0001)$ by -2.9 IQ points per year (s.e. $=0.32,95 \%$ CI -3.56 to -2.30 ). Verbal IQ also declined significantly $(P<0.0001)$ by -2.3 points per year (s.e. $=0.38,95 \%$ CI -3.01 to -1.50 ), as did PIQ $(P=0.015)$ by -1.1 points per year (s.e. $=0.44,95 \% \mathrm{CI}-1.96$ to -0.18$)$. There was a more severe cognitive decline in girls than in boys for FSIQ $(P=0.02$, s.e. $=2.85,95 \%$ CI 1.1 to 12.49$)$ and PIQ $(P=0.019$, s.e. $=2.78,95 \%$ CI 1.16 to 12.26$)$. This difference was not found for VIQ $(P=0.07$, s.e. $=2.96,95 \%$ CI -0.47 to 11.4 ). Figure 1 shows the development of FSIQ, VIQ and PIQ from age 5.5 years to 9.5 years based on the results of the multilevel analyses.

The proportion of children with a familial deletion in the group $(6 \%)$ was comparable to other published results $(11 \%$; $\left.\chi_{1}^{2}=1.65, P=0.20\right),{ }^{30}$ and showed no significant difference in FSIQ, VIQ or PIQ at any age when compared with children with a de novo deletion. Cardiac anomalies were found in 31 of 69 children (45\%) and included tetralogy of Fallot, ventricular septal defect and interrupted aortic arch. No significant difference in FSIQ, VIQ or PIQ score at any age was found.

\section{Cognitive development 7.5-9.5 years}

Figure 2 presents the progression of age-normative (scaled) subtest scores per subtest from age 7.5 years to 9.5 years $(n=44)$. Within the VIQ scale significant declines were found for the subtests Arithmetic $(P=0.008,95 \%$ CI -1.77 to -0.28$)$, Vocabulary $(P<0.0001,95 \%$ CI -2.29 to -0.97$)$ and Comprehension $(P<0.0001,95 \%$ CI -2.07 to -0.77$)$. Within the PIQ scale significant declines were found for the subtest Block Design $(P=0.035,95 \% \mathrm{CI}-1.48$ to -0.06$)$. See online Table DS1.

\section{Change in raw test scores 7.5-9.5 years}

Among the group assessed with the WISC-III-NL at both 7.5 years and 9.5 years of age $(n=29)$ were children who obtained a lower absolute (raw) score at 9.5 years for the same series of questions or tasks that had been presented at age 7.5 years (Fig. 3). A lower raw score implies a decrease in performance where normally an increase would be expected due to ageing and maturation of the child. In a substantial subgroup of 10 children (34\%), this absolute decline in abilities was observed for two or more subtests (online Table DS2). Furthermore, Fig. 3 shows clearly the area in which the children were growing into deficit: their raw scores were better than they were at age 7.5 years, but did not meet

\begin{tabular}{|c|c|c|c|}
\hline & \multicolumn{3}{|c|}{ Age level } \\
\hline & 5.5 years & 7.5 years & 9.5 years \\
\hline Age at assessment, years: mean (s.d.) & $5.5(0.2)$ & $7.5(0.2)$ & $9.5(0.1)$ \\
\hline$n$ & 53 & 64 & 49 \\
\hline Female, $n(\%)$ & $35(66)$ & $43(67)$ & $29(59)$ \\
\hline Familial deletion, $n(\%)$ & $4(8)$ & $3(5)$ & $3(6)$ \\
\hline \multicolumn{4}{|l|}{ IQ scores, mean (s.d.) } \\
\hline Full Scale IQ & $78.8(14.4)$ & $73.5(11.5)$ & $69.1(12.2)$ \\
\hline Girls & $82.1(13.8)$ & $75.4(11.6)$ & $71.6(11.9)$ \\
\hline Boys & $72.6(13.6)^{\mathrm{a} *}$ & $67.3(11.3)^{a_{\star *}}$ & $65.4(11.8)$ \\
\hline Verbal IQ & $80.1(14.2)$ & $76.4(12.3)$ & $71.4(12.8)$ \\
\hline Girls & $82.8(14.8)$ & 77.9 (11.9) & $73.7(12.7)$ \\
\hline Boys & $72.2(8.7)^{a_{\star}}$ & $73.3(12.6)$ & $68.0(12.4)$ \\
\hline Performance IQ & $77.0(14.3)$ & $75.0(12.1)$ & $72.3(12.2)$ \\
\hline Girls & $78.9(15.1)$ & $77.2(12.1)$ & $74.8(11.8)$ \\
\hline Boys & $70.4(8.6)$ & $70.3(10.8)^{\mathrm{a} *}$ & $68.7(12.2)$ \\
\hline
\end{tabular}




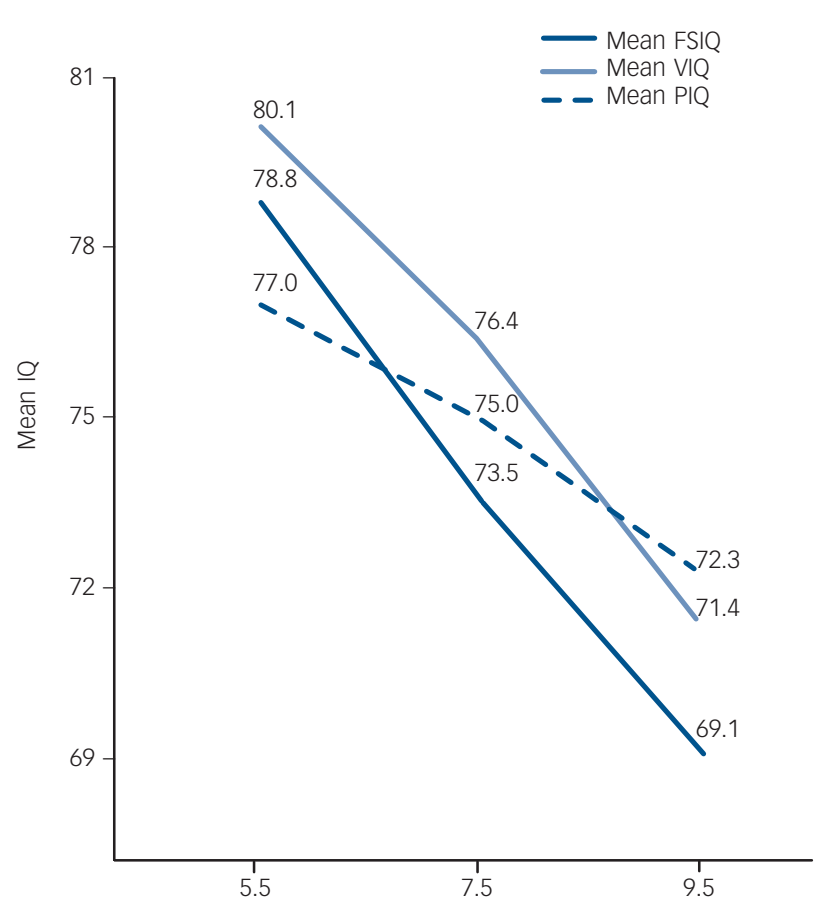

Age at assessment, years

Fig. 1 Development of mean IQ scores in children with the 22q11.2 deletion syndrome from age 5.5 years to 9.5 years (based on results of multilevel analysis; $n=69$ ). FSIQ, Full Scale IQ; PIQ, performance IQ; VIQ, verbal IQ.

the age-related increase required and seen in their peers without the deletion syndrome. Some children showed enough progress in two or more subtests to obtain the same age-specific normative score at age 9.5 years as they did at 7.5 years (Table DS2). Further analysis demonstrated that an absolute decline in two or more subtests was significantly associated with a decreased probability of stabilisation in the normative score in the remaining subtests (Fisher's exact test, $P=0.04$ ).
Comparing the subgroup of children $(n=10)$ with cognitive decline in two or more subtests with the remainder of the participants $(n=19)$ showed that there was no worsening in behaviour in the decline group as measured by changes in CBCL scores $(P=0.85,0.36$ and 0.15 respectively for total, internalising and externalising problem scores). The CBCL results are presented in more detail in online Tables DS3 and DS4.

\section{Possible inter-test effects}

At age 5.5 years the SON-R 2.5-7 $(n=15)$ and the WPPSI-R $(n=41)$ FSIQ scores were not significantly different $(P=0.45)$. At 7.5 years a trend towards a significant difference between FSIQ scores measured by WISC-RN and WISC-III-NL was found $(P=0.073)$. This was to be expected and is in concordance with results described in the WISC-III-NL manual. ${ }^{28}$ Indeed, a larger (and significant) difference in VIQ (10.1 points, $P=0.006)$ was found, with the WISC-RN yielding the higher scores. Performance IQ scores were not significantly different between WISC-RN and WISC-III-NL $(P=0.47)$ at 7.5 years. However, regarding the longitudinal development of cognitive performance, and therefore of particular relevance to the current study, no significant difference was found for the mean change between 7.5 years and 9.5 years in FSIQ, VIQ or PIQ scores as measured by the WISC-RN and WISC-III-NL respectively (online Table DS5). These results suggest that the identified changes in IQ scores were not significantly affected by the cognitive tests used. Nevertheless, to exclude any bias due to the use of different test versions, only WISC-III-NL results were used to analyse the longitudinal course of the raw scores. Parental level of education was not found to be a predictor of cognitive delay (data not shown).

\section{Discussion}

On average, cognitive abilities as expressed by FSIQ scores declined significantly by a mean of 9.7 IQ points between the three assessments at ages 5.5 years, 7.5 years and 9.5 years in this population. Consistent with previous reports, this decline was twice as great for VIQ as it was for PIQ. Within the VIQ scale, significant declines were found for the subtests Vocabulary,

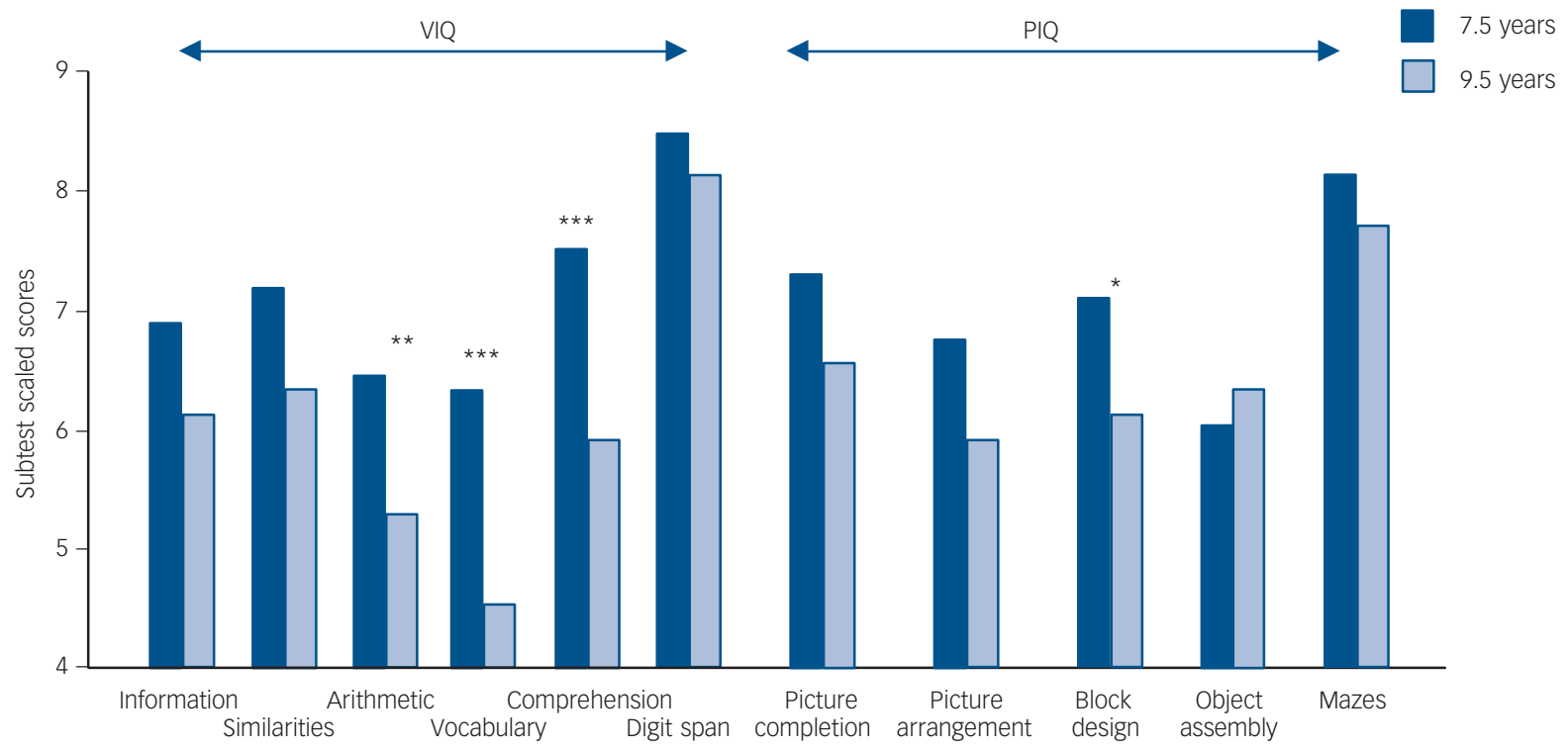

Fig. 2 Change in subtest scaled scores from age 7.5 years to 9.5 years $(n=44)$ as assessed with the Dutch versions of the Wechsler Intelligence Scale for Children - Revised and the Wechsler Intelligence Scale for Children Third Edition. PIQ, performance IQ; VIQ, verbal IQ. ${ }^{*} P<0.05, * * P<0.01, * * * P<0.001$. 


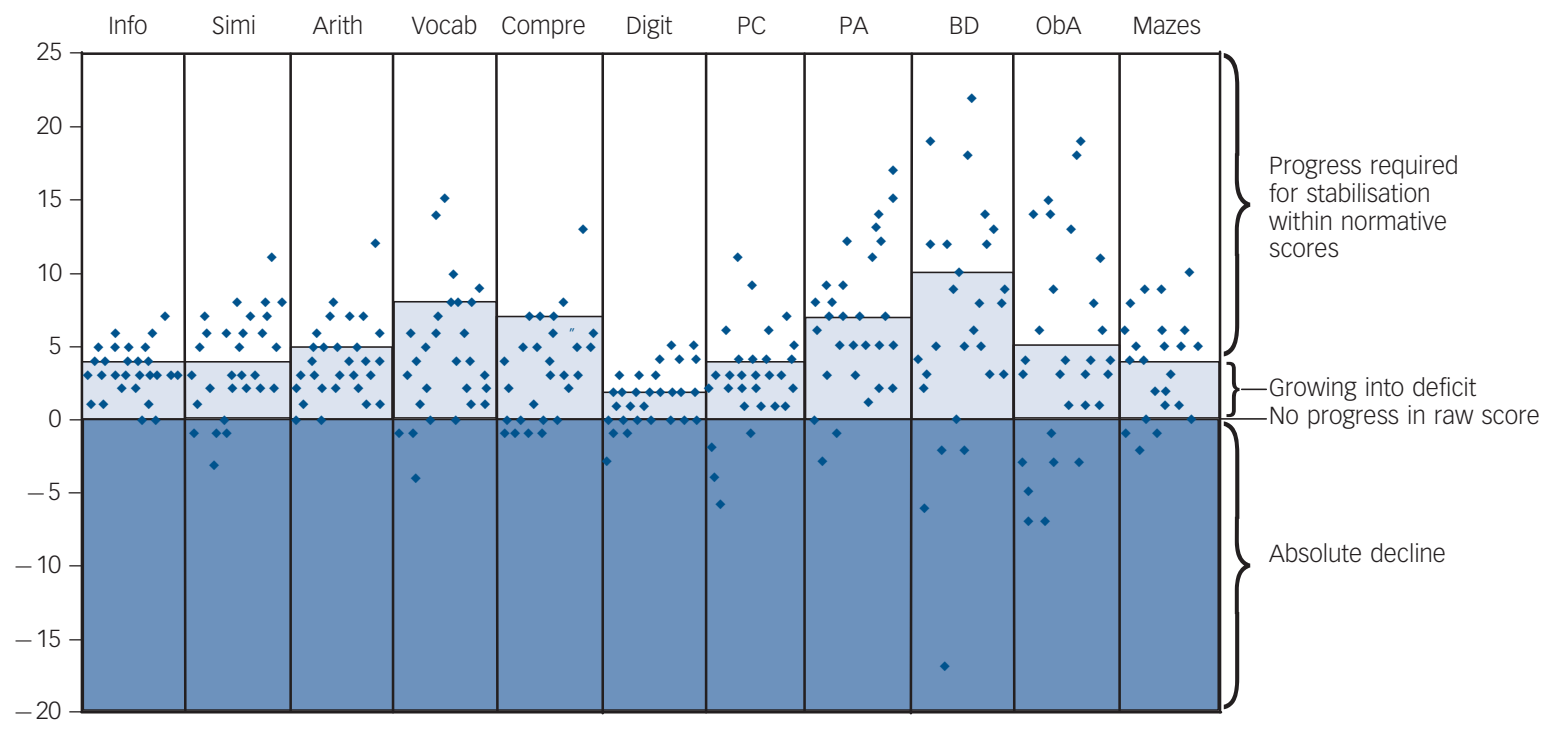

Fig. 3 Progression in raw Wechsler Intelligence Scale for Children (WISC-III) subtest scores between ages 7.5 years and 9.5 years $(n=29)$ expressed as the delta raw score (raw score at 9.5 years minus raw score at 7.5 years).

In the lighter shaded area scores indicate an absolute progress which is, however, not enough to keep up with the normatively required progress. Note that scores in this area, although improving, contribute to a decrease in scaled subtest scores, and ultimately in total IQ score at age 9 . Thus, this area can be considered consistent with the concept of 'growing into deficit'. Raw scores above this area contribute to a stable or improved subtest score at age 9 . Scores in the darker shaded area indicate an absolute decline, implying that the child performed less well in the exact same subtest regardless of age norms. Arith, arithmetic; BD, block design; Compre, comprehension; Digit, digit span; Info, information; $\mathrm{ObA}$, object assembly; PA, picture arrangement; PC, picture completion; Simi, similarities; Vocab, vocabulary.

Comprehension and Arithmetic, suggesting that the overall cognitive decline is mainly driven by a progressive delay in verbal comprehension and expression. Within the scale measuring PIQ, a significant decline was found for the subtest Block Design. Furthermore, an absolute decline in cognitive performance at age 9.5 years as manifested by lower subtest raw scores for at least two subtests (when compared with the same subtests used at age 7.5 years) was found in 10 of the 29 children who were tested twice with the WISC-III-NL. These results indicate that the cognitive decline cannot be fully explained by an inability of the children to keep up with the required age- related increase of raw scores in the cognitive test, a phenomenon also known as growing into deficit. Rather, they indicate the possibility of cognitive deterioration.

The foremost relevance of the results of the current study is that they strongly suggest the possibility that one or more genes at the 22q11.2 locus are contributing to an early cognitive decline as measured by repeated standardised IQ testing. In this regard there may be several plausible candidate genes, including catechol-O-methyltransferase $(C O M T)$, proline dehydrogenase (oxidase) $1(P R O D H)$, phosphatidylinositol 4-kinase, catalytic, alpha (PI4KA) and T-box 1 (TBX1). ${ }^{11,31-33}$ Comprehensive reviews of 22q11.2 genes with potential to influence cognitive development are provided elsewhere. ${ }^{34,35}$ Hence, future genetic studies focusing on the 22q11.2 region may elucidate one of the genetic factors involved in this phenotype.

\section{Implications of cognitive decline}

Early cognitive deficits and academic decline, occurring before the onset of the first psychosis, are frequently reported in schizophrenia and therefore considered an important potential aspect of the schizophrenia phenotype. ${ }^{14,36}$ It could be speculated that the observed decline in this study is likely to be the first symptom of schizophrenic disorder in 22q11.2 deletion syndrome. Interestingly, the absolute decline did not occur in all the children, but rather in a subgroup, parallel to the observation that only a subgroup of people with 22q11.2 deletion syndrome will develop schizophrenia. However, in order to answer this question, follow-up into adulthood is required as the participants in our study are currently too young to be ascertained for the emergence of schizophrenia. Our clinical test protocol ensures that all children have psychiatric assessments from the age of 11 years (at the end of primary school) and earlier if thought necessary by parents or professionals.

The finding of a cognitive decline, mainly driven by a decrease in VIQ, is consistent with the findings of previous (mostly crosssectional) studies in people with 22q11.2 deletion syndrome. Also, the verbal and performance subscales that appear to be most affected are almost entirely consistent with results of previous studies. ${ }^{8,20,37}$ The cross-sectional IQ data of this study show gender differences in favour of girls; in other words, boys are more cognitively affected than girls, and this is already evident at the age of 5.5 years. This is consistent with some findings, ${ }^{38,39}$ yet inconsistent with others that found no gender difference. ${ }^{30}$ Our results, however, also show that girls undergo a more severe cognitive decline than boys between the assessments at ages 5.5, 7.5 and 9.5 years. This is consistent with another recent longitudinal study in older children. ${ }^{12}$ It could be argued that this finding is actually the result of a floor effect of the WISC-III: boys simply cannot decline more severely because the test does not allow for it.

A number of children showed adequate progress in their performance on various subtests: that is, their subtest performance improved beyond the minimum raw score required to maintain the same age-specific normative score. In light of these findings, one could reason that the observed absolute decline in subtest performance is the result of stochastic events rather than being indicative of an underlying process in a subgroup of children. In that case one would expect a random distribution of subtest raw scores showing progress and those showing an absolute decline in the children. However, further analysis demonstrated that an 
absolute decline in two or more subtests was significantly associated with a decreased probability of progress in the remaining subtests, thus refuting stochastic variability in performance as an explanation of the findings. In other words, the results strongly suggest the existence of a subgroup of children with the deletion syndrome who show a marked decline in cognitive abilities, including an absolute decline in performance on two or more subtests.

\section{Limitations of the study}

The use of different cognitive tests at various ages can be considered a limitation of this study. Two different approaches were used to overcome this problem. First, additional statistical analyses were performed to rule out any test-specific effects of IQ scores with regard to both the cross-sectional and the longitudinal (IQ change) data. Except for a significant difference in cross-sectional VIQ scores between the WISC-RN and the WISC-III-NL, a well-known distinguishing characteristic of these scales, no difference was found in relation to the different tests. More importantly, the change in IQ score was not significantly affected by the use of the different tests. In addition, the second approach to rule out test-related effects consisted of the analysis of a subgroup of patients who were tested twice with exactly the same test (WISC-III-NL). Findings of this analysis confirmed the cognitive decline, and in addition indicated the absolute loss of abilities in a subgroup of patients. The small size of this subgroup $(n=10)$ did not provide sufficient power to examine the possibility of a distinguishing cognitive profile.

Another limitation of our study is that neuropsychiatric assessment of these young children was not part of our test protocol. Therefore, we cannot evaluate whether the observed changes in IQ were correlated with psychiatric diagnoses such as attention-deficit hyperactivity disorder and autism-spectrum disorder. However, previous studies by different groups strongly suggest that such a correlation either does not exist, or is likely to be of modest strength at most. ${ }^{4,39,40}$

\section{Clinical relevance}

Apart from relevance to the understanding of the genetic underpinnings of cognitive deterioration, the observed decline also has clinical consequences for individuals with $22 \mathrm{q} 11.2$ deletion syndrome. The results stress the importance of early screening and continuous monitoring of the cognitive development of children with this syndrome. Over the years some of these children may be increasingly challenged beyond their cognitive capabilities, but are expected to function at the same academic level they were able to meet previously. A chronic situation of too much stress is a known risk factor for a range of internalising and externalising psychiatric disorders.

In conclusion, the results of this longitudinal study into the cognitive development of young children with 22q11.2 deletion syndrome show a progressive decrease in FSIQ score starting as early as 5.5 years of age, mainly driven by a declining VIQ. The decline appears as an average group effect, but not all children are (equally) affected. In part the results show that this decline occurs as a result of stagnation of cognitive development relative to increasing cognitive requirements. However, there appears to be a subgroup of children in whom this decline is characterised by an absolute loss of cognitive faculties.

\section{Future research}

From a research perspective the 22q11.2 deletion can be viewed as a unique experiment of nature providing a powerful model in which the same genetic variant is associated with a highly increased risk of developing brain-related phenotypes. Elucidating these various 22q11.2 deletion syndrome-related phenotypes, as well as identifying the underlying genetic mechanism that can account for them, may provide important novel insights in neuroscience. The findings reported here suggest the possibility that one or several genes at the 22q11.2.2 locus contribute to an early cognitive decline.

Sasja N. Duijff, MSC, Petra W. J. Klaassen, MSC, Henriette F. N. Swanenburg de Veye, PhD, Department of Pediatric Psychology, Frits A. Beemer, PhD, Department of Medical Genetics, Gerben Sinnema, PhD, Department of Pediatric Psychology, University Medical Centre Utrecht, Utrecht; Jacob A. S. Vorstman, PhD, Department of Child and Adolescent Psychiatry and the Rudolf Magnus Institute of Neurosciences, University Medical Centre Utrecht, Utrecht, The Netherlands

Correspondence: Sasja N. Duijff, University Medical Centre Utrecht, Department of Paediatric Psychology, KA.00.004.0, PO Box 85090, 3508 AB Utrecht, The Netherlands. Email: S.Duijff@umcutrecht.nl

First received 30 May 2011, final revision 1 Sep 2011, accepted 11 Nov 2011

\section{Funding}

The study was sponsored by a grant from Stichting Nuts Ohra (SNO-T-03-32).

\section{Acknowledgements}

We thank all the participating children and parents. We also wish to thank Paul Westers, $\mathrm{PhD}$, for statistical advice.

\section{References}

1 Gothelf D. Velocardiofacial syndrome. Child Adolesc Psychiatr Clin N Am 2007; 16: 677-93.

2 Kates WR, Antshel KM, Fremont WP, Shprintzen RJ, Strunge LA, Burnette CP, et al. Comparing phenotypes in patients with idiopathic autism to patients with velocardiofacial syndrome (22q11 DS) with and without autism. Am J Med Genet A 2007; 143: 2642-50.

3 Murphy KC, Jones LA, Owen MJ. High rates of schizophrenia in adults with velo-cardio-facial syndrome. Arch Gen Psychiatry 1999; 56: 940-5.

4 Vorstman JA, Morcus ME, Duijff SN, Klaassen PW, Heineman-de Boer JA Beemer FA, et al. The 22q11.2 deletion in children: high rate of autistic disorders and early onset of psychotic symptoms. J Am Acad Child Adolesc Psychiatry 2006; 45: 1104-13.

5 Gothelf D, Frisch A, Munitz H, Rockah R, Laufer N, Mozes T, et al. Clinical characteristics of schizophrenia associated with velo-cardio-facial syndrome. Schizophr Res 1999; 35: 105-12.

6 Golding-Kushner KJ, Weller G, Shprintzen RJ. Velo-cardio-facial syndrome: language and psychological profiles. Craniofac Genet Dev Biol 1985; 5: 259-66.

7 Gothelf D, Aviram-Goldring A, Burg M, Steinberg T, Mahajnah M, Frisch A, et al. Cognition, psychosocial adjustment and coping in familial cases of velocardiofacial syndrome. J Neural Transm 2007; 114: 1495-501.

8 Green T, Gothelf D, Glaser B, Debbane M, Frisch A, Kotler M, et al. Psychiatric disorders and intellectual functioning throughout development in velocardiofacial (22q11.2 deletion) syndrome. J Am Acad Child Adolesc Psychiatry 2009; 48: 1060-8.

9 Niklasson L, Gillberg C. The neuropsychology of 22q11 deletion syndrome. A neuropsychiatric study of 100 individuals. Res Dev Disabil 2010; 31: 185-94.

10 Shprintzen RJ. Velo-cardio-facial syndrome: a distinctive behavioral phenotype. Ment Retard Dev Disabil Res Rev 2000; 6: 142-7.

11 Gothelf D, Eliez S, Thompson T, Hinard C, Penniman L, Feinstein C, et al. COMT genotype predicts longitudinal cognitive decline and psychosis in 22q11.2 deletion syndrome. Nat Neurosci 2005; 8: 1500-2.

12 Antshel KM, Shprintzen R, Fremont W, Higgins AM, Faraone SV, Kates WR. Cognitive and psychiatric predictors to psychosis in velocardiofacial syndrome: a 3-year follow-up study. J Am Acad Child Adolesc Psychiatry 2010; 49: 333-44.

13 Flynn JR. Massive IQ gains in 14 nations: what IQ tests really measure. Psychol Bull 1987; 101: 171-91. 
14 Woodberry KA, Giuliano AJ, Seidman LJ. Premorbid IQ in schizophrenia: a meta-analytic review. Am J Psychiatry 2008; 165: 579-87.

15 Bilder RM, Reiter G, Bates J, Lencz T, Szeszko P, Goldman RS, et al. Cognitive development in schizophrenia: follow-back from the first episode. J Clin Exper Neuropsychol 2006; 28: 270-82.

16 Fuller R, Nopoulos P, Arndt S, O'Leary D, Ho BC, Andreasen NC. Longitudina assessment of premorbid cognitive functioning in patients with schizophrenia through examination of standardized scholastic test performance. Am J Psychiatry 2002; 159: 1183-9.

17 Van Oel CJ, Sitskoorn MM, Cremer MPM, Kahn RS. School performance as a premorbid marker for schizophrenia: a twin study. Schizophr Bull 2002; 28 : 401-14.

18 Monte RC, Goulding SM, Compton MT. Premorbid functioning of patients with first-episode nonaffective psychosis: a comparison of deterioration in academic and social performance, and clinical correlates of Premorbid Adjustment Scale scores. Schizophr Res 2008; 104: 206-13.

19 Debbané M, Glaser B, David MK, Feinstein C, Eliez S. Psychotic symptoms in children and adolescents with 22q11.2 deletion syndrome: neuropsychological and behavioral implications. Schizophr Res 2006; 84: 187-93.

20 Gothelf D, Feinstein C, Thompson T, Gu E, Penniman L, Van Stone E, et al. Risk factors for the emergence of psychotic disorders in adolescents with 22q11.2 deletion syndrome. Am J Psychiatry 2007; 164: 663-9.

21 Vorstman JA, Jalali GR, Rappaport EF, Hacker AM, Scott C, Emanuel BS. MLPA: a rapid, reliable, and sensitive method for detection and analysis of abnormalities of 22q. Hum Mutat 2006; 27: 814-21.

22 American Psychiatric Association. Diagnostic and Statistical Manual of Mental Disorders (4th edn) (DSM-IV). APA, 1994.

23 UNESCO. ISCED 1997. UNESCO Institute for Statistics, 2006.

24 Centraal Bureau voor de Statistiek. Jaarboek Onderwijs In Cijfers 2006: 85-7. CBS, 2005.

25 Vander Steene G, Bos A. WPPSI-R Wechsler Preschool and Primary Scale of Intelligence, Vlaams-Nederlandse Aanpassing voorlopige versie, Handleiding. Swets \& Zeitlinger, 1997.

26 Tellegen PJ, Winkel M, Wijnberg-Williams BJ, Laros JA. Snijders-Oomen Non-verbal Intelligence Test Revised (SON-R 21/2-7): Manual and Research Report. Hogrefe Uitgevers, 1998.

27 Vander Steene G, van Haassen PP, de Bruyn EEJ, Coetsier P, Pijl YJ, Poortinga $\mathrm{YH}$, et al. WISC-R: Nederlandstalige Uitgave. Swets \& Zeitlinger, 1986.
28 Wechsler D, Kort W, Schittekatte M, Dekker PH, Verhaege P, Compaan EL, et al. WISC Intelligence Scale for Children Third Edition NL. Harcourt Assessment, 2005

29 Achenbach TM, Rescorla LA. Manual for the ASEBA School-Age Forms \& Profiles. University of Vermont Research Center for Children, Youth, \& Families, 2001.

30 Desmedt B, Devriendt K, Fryns JP, Vogels A, Gewillig M, Swillen A. Intellectual abilities in a large sample of children with velo-cardio-facial syndrome: an update. J Intellect Disabil Res 2007; 51: 666-70.

31 Raux G, Bumsel E, Hecketsweiler B, van Amelsvoort T, Zinkstok J, Manouvrier-Hanu $S$, et al. Involvement of hyperprolinemia in cognitive and psychiatric features of the 22q11 deletion syndrome. Hum Mol Genet 2007; 16: 83-91.

32 Vorstman JAS, Chow EW, Ophoff RA, van Engeland H, Beemer FA, Kahn RS, et al. Association of the PIK4CA schizophrenia-susceptibility gene in adults with the 22q11.2 deletion syndrome. Am J Med Genet B 2009; 150: 430-3.

33 Paylor R, Glaser B, Mupo A, Ataliotis P, Spencer C, Sobotka A, et al. Tbx1 haploinsufficiency is linked to behavioral disorders in mice and humans: implications for 22q11 deletion syndrome. Proc Natl Acad Sci USA 2006; 103: 7729-34.

34 Karayiorgou M, Simon TJ, Gogos JA. 22q11.2 microdeletions: linking DNA structural variation to brain dysfunction and schizophrenia. Nat Rev Neurosci 2010; 11: 402-16.

35 Williams NM. Molecular mechanisms in 22q11 deletion syndrome. Schizophr Bull 2011; 37: 882-9.

36 Kremen WS, Buka SL, Seidman LJ, Goldstein JM, Koren D, Tsuang MT. IQ decline during childhood and adult psychotic symptoms in a community sample: a 19-year longitudinal study. Am J Psychiatry 1998; 155: 672-7.

37 Gothelf D, Penniman L, Gu E, Eliez S, Reiss AL. Developmental trajectories of brain structure in adolescents with 22q11.2 deletion syndrome: a longitudinal study. Schizophr Res 2007; 96: 72-81.

38 Antshel KM, Abdulsabur N, Roizen N, Fremont W, Kates WR. Sex differences in cognitive functioning in velocardiofacial syndrome (VCFS). Dev Neuropsychol 2005; 28: 849-69.

39 Niklasson L, Rasmussen P, Oskarsdottir S, Gillberg C. Autism, ADHD, mental retardation and behavior problems in 100 individuals with 22 q11 deletion syndrome. Res Dev Disabil 2009; 30: 63-73.

40 Jansen PW, Duijff SN, Beemer FA, Vorstman JA, Klaassen PW, Morcus ME, et al. Behavioral problems in relation to intelligence in children with 22q11.2 deletion syndrome: a matched control study. Am J Med Genet A 2007; 143: 574-80. 